\title{
Fabrication of alginate-based hydrogel cross-linked via horseradish peroxidase for articular cartilage engineering
}

Sepideh Saghati ${ }^{1}$, Ali Baradar Khoshfetrat ${ }^{2 *}{ }^{*}$, Hamid Tayefi Nasrabadi ${ }^{1}$, Leila Roshangar ${ }^{1}$ and Reza Rahbarghazi ${ }^{3,4^{*}+}$ (D)

\begin{abstract}
Objective: We aimed to detect the effect of a couple of parameters including Alg, $\mathrm{H}_{2} \mathrm{O}_{2}$, and $\mathrm{HRP}$ concentrations on the gelation time of Alg-based hydrogels using an enzymatic cross-linked procedure.

Results: NMR, UV-Vis, and ATR-FTIR analyses confirmed the conjugation of Ph to the Alg backbone. Data showed gelation time was delayed with the increase and reduction of $\mathrm{H}_{2} \mathrm{O}_{2}$ and HRP, respectively. We noted that hydrogel consisted of 1.2\% (w/v) Alg, $5 \cup \mathrm{HRP}$, and $100 \mathrm{mM} \mathrm{H}_{2} \mathrm{O}_{2}$ yielded an appropriate gelation time with appropriate mechanical properties. The addition of $0.5 \%(\mathrm{v} / \mathrm{v})$ Col developed hydrogel increased the gelation time. The data showed that Alg, HRP, and $\mathrm{H}_{2} \mathrm{O}_{2}$ with the ratio of 1:0.54:0.54 had proper physicochemical features for cartilage engineering.
\end{abstract}

Keywords: Alginate, Hydrogel, Phenolation, Enzymatic cross-linking

\section{Introduction}

In non-vascular tissues such as articular cartilage, a limited self-renewal capacity leads to delayed healing procedures. As a correlate, the development of novel therapeutic approaches for the acceleration of healing procedures in injured articular cartilage is at the center of attention [1]. Hydrogels with improved mechanical and biological properties can mechanically and biologically regulate cell bioactivity, making them suitable compounds for in vitro and in vivo conditions [2-4]. Among hydrogel polymers, natural polymers such as

\footnotetext{
*Correspondence: khoshfetrat@sut.ac.ir; rezarahbardvm@gmail.com; rahbarghazir@tbzmed.ac.ir

${ }^{\dagger}$ Ali Baradar Khoshfetrat and Reza Rahbarghazi contributed equally to this work

${ }^{2}$ Chemical Engineering Faculty, Sahand University of Technology,

51335-1996 Tabriz, Iran

${ }^{4}$ Department of Applied Cell Sciences, Faculty of Advanced Medical Sciences, Tabriz University of Medical Sciences, 5154853431 Tabriz, Iran Full list of author information is available at the end of the article
}

Alg, unbranched binary copolymers of (1-4)-linked $\beta$-Dmannuronic and $\alpha$-L-guluronic residues have been used as cartilage regenerative substrates $[5,6]$. Both ionic and enzymatic cross-linking procedures are suitable for the fabrication of Alg-based hydrogels by anionic gelation $\left(\mathrm{CaCl}_{2}\right)$ and enzymatic crosslinking using phenolic groups $[7,8]$. Because of ion exchanging in ionic crosslinked hydrogels, the final structures lack appropriate mechanical properties [9]. Several advantages such as mild reaction conditions (ear physiological $\mathrm{pH}$ and temperature) and controllable mechanical properties have doubled the importance of enzymatically cross-linked hydrogels in the fabrication of engineered scaffolds [10]. In support of this notion, Alg-based hydrogels are suitable scaffolds in the field of tissue engineering. Using different synthesis protocols and modalities, it is possible to synthesize Alg hydrogels with distinct mechanical properties comparable to the in vivo conditions [11]. Natural polymers like $\mathrm{Col}$ benefits from the advantages of having cell-recognizable moieties, hydrated 3D networks, 
lack of immunogenicity, and stimulates the cells to produce ECM [12]. Despite these superiorities, decreased mechanical stability of Col limits its extensive biological application. To bypass this obstacle, several studies have tried to combine Col with other natural components such as polysaccharides like Alg to obtain a suitable scaffold with controllable mechanical and chemical properties [13]. HRP is one of the applicable enzymes for cross-linking. $\mathrm{HRP}$ can react with $\mathrm{H}_{2} \mathrm{O}_{2}$ when added to the solution containing a polymer-phenol mixture [14]. The gelation time of the hydrogels is one of the most important factors that must be considered in controlling the final physicochemical and mechanical properties. This value depends on the reaction condition like temperature, components concentrations, cross-linking density, and the extent of Ph groups $[15,16]$. Here, we aimed to synthesize hydrogel of Alg-based hydrogel with $\mathrm{Ph}$ groups (Alg-Ph) using the HRP-mediated cross-linking and evaluate the reaction conditions on the gelation time. The close association with hydrogel gelation time and concentrations of Alg, HRP, and $\mathrm{H}_{2} \mathrm{O}_{2}$ was detected. This hydrogel was designed in line with cartilage tissue engineering. Previously, we showed the chondrogenic properties of Alg-Ph-based hydrogels in the orientation of human mesenchymal stem cells toward chondrocytelike cells in in vitro conditions [17]. Here, we tried to present improved formulation (time and concentration of components) and procedure required for an appropriate HRP-mediated cross-linked Alg-Ph hydrogel.

\section{Main text \\ Materials and methods \\ Materials}

Bovine cutaneous type I Col and HRP (P8375-1KU) were obtained from Sigma-Aldrich. The activity of HRP is based on the pyrogallol unit. Sodium Alg (molecular weight: $7 \times 10^{4}$; molar ratio of mannuronic acid to guluronic acid: 0.65) was purchased from KIMICA Corporation (Japan). $\mathrm{H}_{2} \mathrm{O}_{2}$ solution was obtained from Merck. EDC, NHS, MES, acetone, EtOH, and tyramine hydrochloride (Cat no.: T2879) were purchased from Sigma-Aldrich.

\section{Synthesis of Alg-Ph}

To prepare Alg-Ph moieties, Alg was conjugated with tyramine using carbodiimide-mediated condensation [18]. To this end, we prepared $0.2 \%(w / v)$ MES and the $\mathrm{pH}$ value was set to 6 . Alg solution (1\%) was prepared by adding sodium Alg (7.5 g) to the solution. The solution was mixed until Alg completely dissolved. The process was continued by the addition of NHS $(0.85 \mathrm{~g})$, EDC $(2.83 \mathrm{~g})$, and tyramine hydrochloride $(5.88 \mathrm{~g})$ to the mixture. When the constituents were entirely dissolved after
$20 \mathrm{~h}$ at $25^{\circ} \mathrm{C}$, the $\mathrm{pH}$ was changed to 8.6 (Fig. 1). The synthesized polymer was extracted using acetone, washed with EtOH overnight, and then lyophilized.

\section{Fabrication of hydrogel}

To fabricate hydrogel composed of Alg- $\mathrm{Ph} / \mathrm{Col}$, Alg$\mathrm{Ph}$ powder was mixed with the $0.5 \% \mathrm{Col}(\mathrm{w} / \mathrm{v} \%)$ solution (Fig. 1). In this study, different HRP concentrations were used for enzymatic cross-linking of Alg-Ph. HRP was added to the $\mathrm{Al}-\mathrm{Ph} / \mathrm{Col}$ solution and mixed gently. On the other hand, different concentrations of Alg$\mathrm{Ph}$ including $1,1.2,1.5 \% \mathrm{w} / \mathrm{v}$ ) were used to optimize the gelation time. To synthesize a hydrogel with appropriate physical properties, different molarities of $\mathrm{H}_{2} \mathrm{O}_{2}$ overlaid the solution and mixed gently until complete gelation occurred. Then, the prepared hydrogel was maintained at $37^{\circ} \mathrm{C}$ for $4 \mathrm{~h}$. Similar to the protocol used for the synthesis of the Alg- $\mathrm{Ph} / \mathrm{Col}$ hydrogel, the Alg- $\mathrm{Ph}$ hydrogel was also synthesized. From several synthesis protocols, those with suitable physical properties with distinct concentrations were selected for mechanical testing. These samples were named as Alg-Ph1, Alg-Ph2 and Alg-Ph3 and the final concentration of Col, Alg- $\mathrm{Ph}, \mathrm{HRP}$ and $\mathrm{H}_{2} \mathrm{O}_{2}$ was set to $0.5 \%(\mathrm{w} / \mathrm{v}), 1.2 \%(\mathrm{w} / \mathrm{v}), 5$ (units $/ \mathrm{ml}), 100(\mathrm{mM})$, respectively.

\section{Scaffold characterization ATR-FTIR}

Varian 610 spectrometer (Agilent Technologies, USA) was used to detect the ATR-FTIR spectrum of Alg-Ph and $\mathrm{Alg}-\mathrm{Ph} / \mathrm{Col}$ hydrogels using a DTGS detector and a diamond crystal. Hydrogels were fabricated in cylindrical shape then frozen at $-20^{\circ} \mathrm{C}$ for $24 \mathrm{~h}$ and lyophilized.

\section{Gelation time}

Here, we performed gelation time assay in four different steps as follows; First, we prepared hydrogel consisted of $1.2 \%(\mathrm{w} / \mathrm{v}) \mathrm{Alg}-\mathrm{Ph}$ and $100 \mathrm{mM} \mathrm{H}_{2} \mathrm{O}_{2}$ and different concentrations of HRP, including 3, 5, 6, 8, and $10 \mathrm{U}$. Second, $1.2 \%(\mathrm{w} / \mathrm{v}) \mathrm{Alg}-\mathrm{Ph}$ and $5 \mathrm{U}$ HRP were combined and different doses of $\mathrm{H}_{2} \mathrm{O}_{2}$, including $15,25,30,40,50$, and $100 \mathrm{mM}$ were used for gelation; In the next step, different Alg-Ph concentrations $(1,1.2 \%, 1.5 \%)$ were added to the mixture composed of $5 \mathrm{U}$ HRP plus $100 \mathrm{mM} \mathrm{H}_{2} \mathrm{O}_{2}$. After completion of these experiments, we selected hydrogels with appropriate consistency with a short gelation time. We performed the experiments with selected doses of Alg-Ph, HRP, and $\mathrm{H}_{2} \mathrm{O}_{2}$ and $0.5 \%$ (w/v) Col solution.

To assess gelation time, hydrogels composed of Alg-Ph and HRP were placed in culture plates (100-200 $\mu \mathrm{l}$ per 48 -well plates) and gently stirred at $80 \mathrm{rpm}$. Then, $\mathrm{H}_{2} \mathrm{O}_{2}$ solution was added while mixing. We considered the 


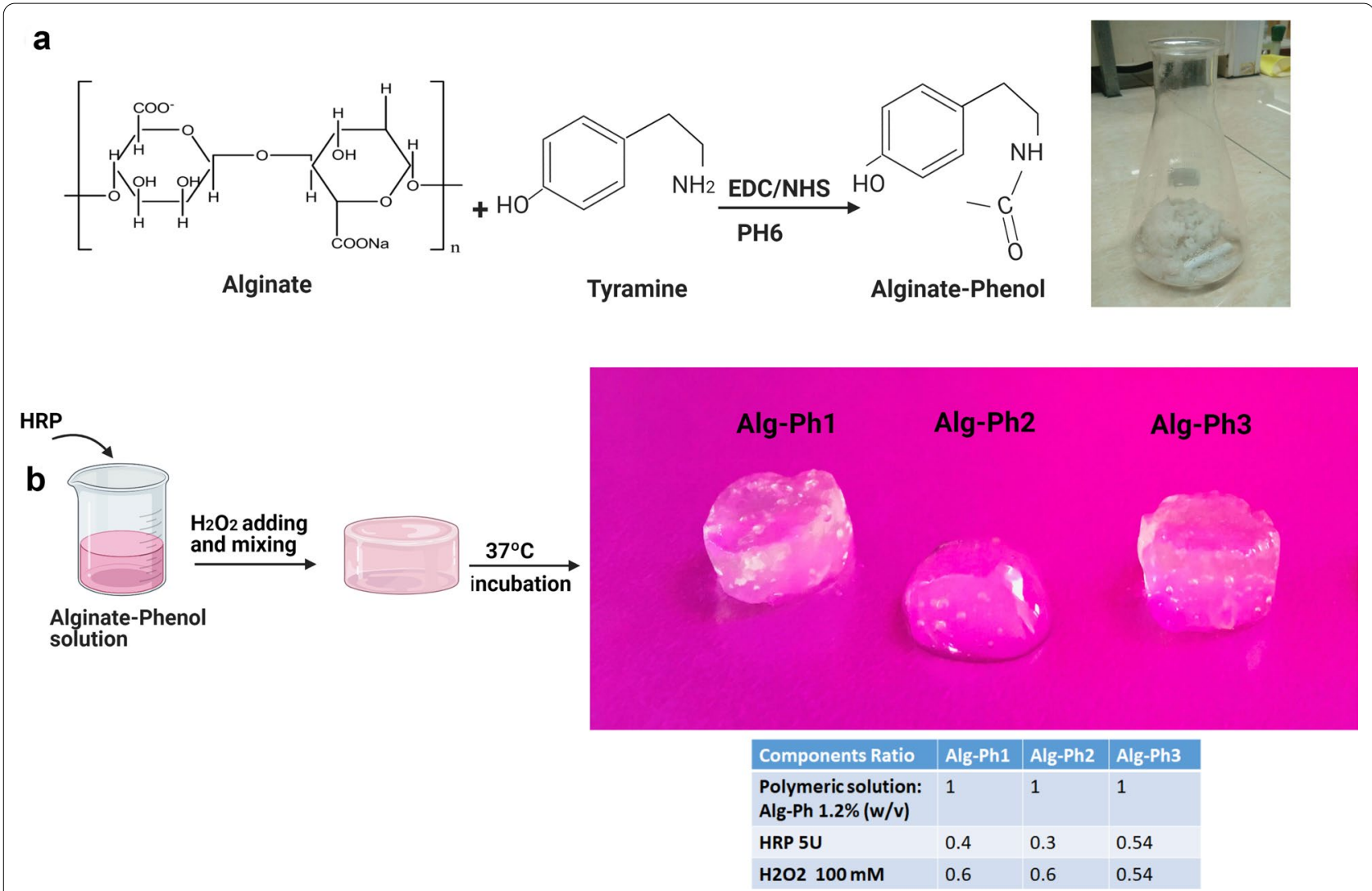

Fig. 1 Schematic illustration of the Alg phenolation process (a), the Alg-Ph hydrogel fabrication (b)

gelation of hydrogels when the stirring was inhibited and led to the solution swelling.

\section{Mechanical testing}

After several tryouts with various Alg, $\mathrm{HRP}$, and $\mathrm{H}_{2} \mathrm{O}_{2}$ concentrations, we indicated that hydrogel consisted of $1.2 \%(\mathrm{w} / \mathrm{v})$ Alg- $\mathrm{Ph}, 5(\mathrm{u} / \mathrm{ml}) \mathrm{HRP}$, and $100(\mathrm{mM}) \mathrm{H}_{2} \mathrm{O}_{2}$ exhibited appropriate physical stability. For mechanical testing, we used three different hydrogels as follows; Alg-Ph-1 [Alg-Ph 1.2\% v/w + HRP $5 \mathrm{U}+\mathrm{H}_{2} \mathrm{O}_{2} 100 \mathrm{mM}$ (1:0.4:0.6)], Alg-Ph-2 [Alg-Ph 1.2\% v/w + HRP $5 \mathrm{U}$ $+\mathrm{H}_{2} \mathrm{O}_{2} 100 \mathrm{mM}$ (1:0.3:0.6)], and Alg-Ph-3 [Alg-Ph 1.2\% $\mathrm{v} / \mathrm{w}+\mathrm{HRP} 5 \mathrm{U}+\mathrm{H}_{2} \mathrm{O}_{2} 100 \mathrm{mM}$ (1:0.54:0.54)]. Zwick/ Roell (Z010, Germany) tensile machine equipped with a $10-\mathrm{kN}$ load cell was used to assess mechanical characterization of cylindrical shaped $(1 \times 1 \mathrm{~cm})$ hydrogels. Testing was carried out at a compression rate of $2 \mathrm{~mm}$ per minute. The compressive modulus of the hydrogels was calculated as the slope of a linear fit to the stress-strain curve over $5-10 \%$ strain. The test was done in triplicate.

\section{SEM imaging}

To this end, the sample was frozen at $-80{ }^{\circ} \mathrm{C}$ for $18 \mathrm{~h}$ followed by lyophilization for $24 \mathrm{~h}$. SEM (JEOL 840 73,
Joel, Japan) was used for the assessment of morphological properties. Samples were sputter-coated and placed on a holder inside the SEM chamber.

\section{Results}

\section{Gelation time}

Here, the effect of Alg-Ph, $\mathrm{H}_{2} \mathrm{O}_{2}$, and HRP concentrations was assessed on the Alg-Ph hydrogel gelation time (Fig. 2a-c). Data showed that the gelation time was reduced by decreasing the concentration of $\mathrm{H}_{2} \mathrm{O}_{2}$ (in the range of 3-10 sunits $/ \mathrm{ml}$ ) and increasing the HRP concentration. We noted an increased gelation time when the concentration of Alg reached 1.5\% (w/v). The existence of $\mathrm{Col}$ in the hydrogel structure increased gelation time (Fig. 2d).

\section{Chemical characterization}

To confirm the chemical conjugation of $\mathrm{Ph}$ groups with the Alg backbone, UV-Vis and NMR analyses were performed. The chemical composition of Alg$\mathrm{Ph}$ and $\mathrm{Alg}-\mathrm{Ph} / \mathrm{Col}$ hydrogels was determined using ATR-FTIR analysis (Fig. 3a). The FTIR spectra indicate the characteristic peaks of $\mathrm{Alg}$ and Col including strong band at $1630-1634 \mathrm{~cm}^{-1}$ proving the $\mathrm{C}=\mathrm{C}$ 

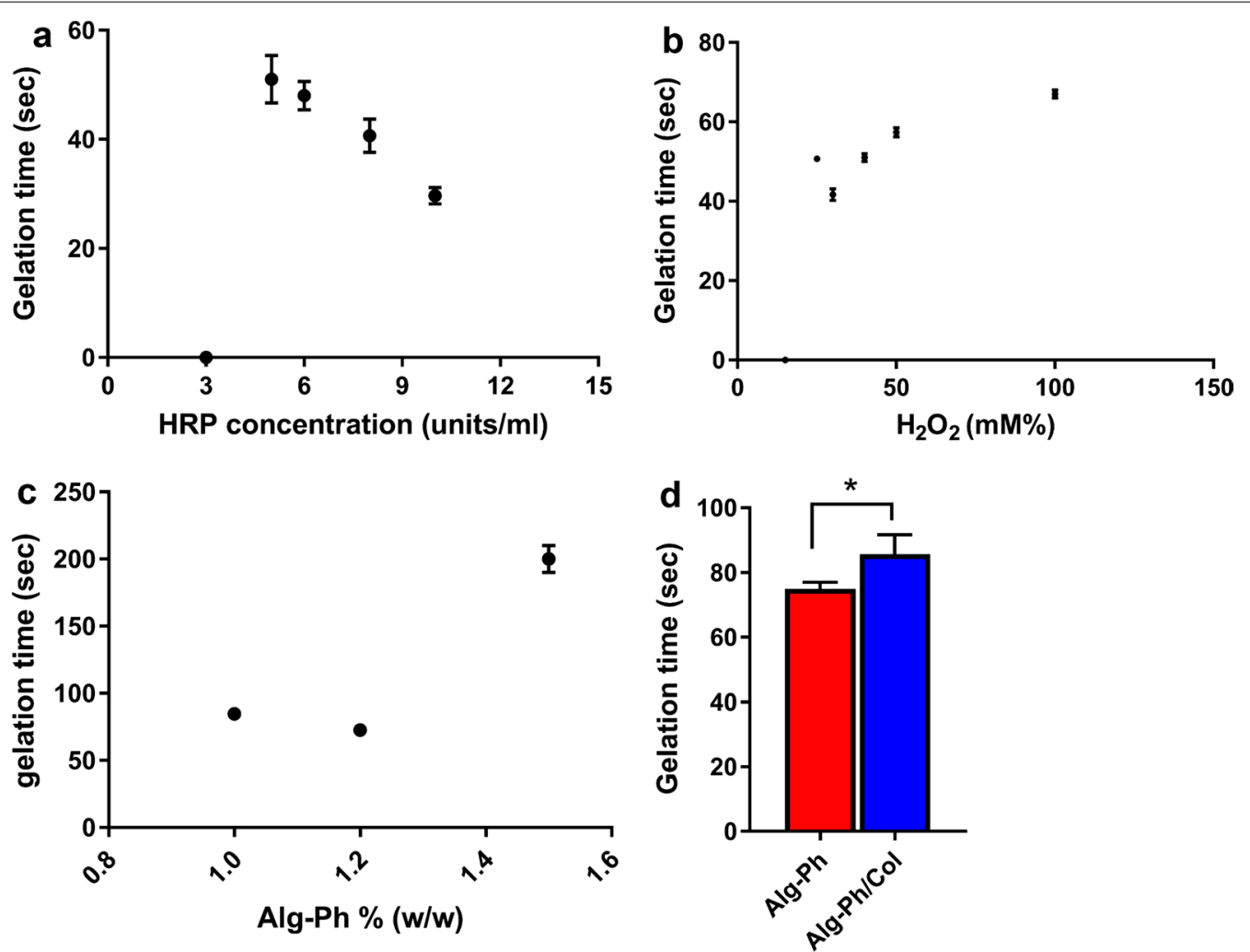

Fig. 2 Association of gelation time for Alg-Ph hydrogels (a) HRP concentration (Alg-Ph 1.2\% w/v, $\mathrm{H}_{2} \mathrm{O}_{2} 100 \mathrm{mM}$ ), (b) $\mathrm{H} 2 \mathrm{O} 2$ concentration (Alg-Ph $1.2 \% \mathrm{w} / \mathrm{v}, \mathrm{HRP} 5 \cup(\mathbf{c})$ Alg-Ph concentration, and (d) the combination of Alg-Ph with $0.5 \%(\mathrm{w} / \mathrm{v})$ Col. Student $t$ test; $\mathrm{p}<0.05(\mathrm{n}=3)$

groups of tyramine, hydroxyl $\mathrm{O}-\mathrm{H}$ broadband in the range of $3000-3600 \mathrm{~cm}^{-1}$, ether aliphatic $\mathrm{C}-\mathrm{H}$ stretching band $\left(2900-2850 \mathrm{~cm}^{-1}\right)$, and stretching vibrations of carboxylic functional groups related to aliphatic ether $\left(1190 \mathrm{~cm}^{-1}\right)$ [17]. Also, the spectra of Alg-Ph/Col exhibited the Col fingerprint bands at $1260-1270 \mathrm{~cm}^{-1}$ related to the $\mathrm{C}-\mathrm{N}$ and $\mathrm{N}-\mathrm{H}$ bands, $1630 \mathrm{~cm}^{-1}$ (typical of amide I), $3418 \mathrm{~cm}^{-1}$ (amide A), and $2930 \mathrm{~cm}^{-1}$ (amide B). According to the NMR analysis, distinguishing peak areas of Ph were observed at 6.6-7.2 ppm (Fig. 3b) [19]. The peaks related to protons on carbons adjacent to a carboxylic acid were detected in the $2-3 \mathrm{ppm}$ region. The spectra represent characteristic peaks of Alg polymer at 4-5 ppm. The UV-Vis spectra of the Alg-Ph solution were detected in an aqueous solution at $275 \mathrm{~nm}$ which is in agreement with the NMR results and associated with Ph groups (Fig. 3c) [20].

\section{Morphological investigations}

SEM imaging showed homogeneous microstructure and improved interconnectivity of Alg- $\mathrm{Ph}$ and Alg-Ph/ Col hydrogels (Fig. 3d). As shown in Fig. 3d, Alg and Col micro-fibrils are recognizable throughout the structure. These data confirm the capability of our methodology to fabricate hydrogels with appropriate pore size and homogenous porosity for cell seeding applications.

\section{Mechanical characterization}

Figure 3e illustrates the compressive mechanical properties of three final selected compositions named Alg-Ph1, Alg-Ph2, and Alg-Ph3. The data showed that the Alg-Ph3 hydrogel had the highest compressive strength among three samples with the ultimate compressive strength of $750(\mathrm{KPa})$ and broke at $49-50 \%$ strain. While the lowest stability was related to the Alg-Ph2 hydrogel broke at $45 \%$ strain with an ultimate compressive strength of $260(\mathrm{KPa})$. According to the data, the highest compressive modulus was exhibited by the Alg-Ph3 hydrogel (13.4 MPa compared with 11.5 MPa for Alg-Ph1 and Alg-Ph2).

\section{Discussion}

Alg-Ph was synthesized via the peroxidase-catalyzed oxidative crosslinking method. The relation between hydrogels gelation time and concentration of components was assessed. We performed different combinations and formulas with various concentrations of Alg, HRP, and $\mathrm{H}_{2} \mathrm{O}_{2}$ to find appropriate gelation time. Gelation time is 


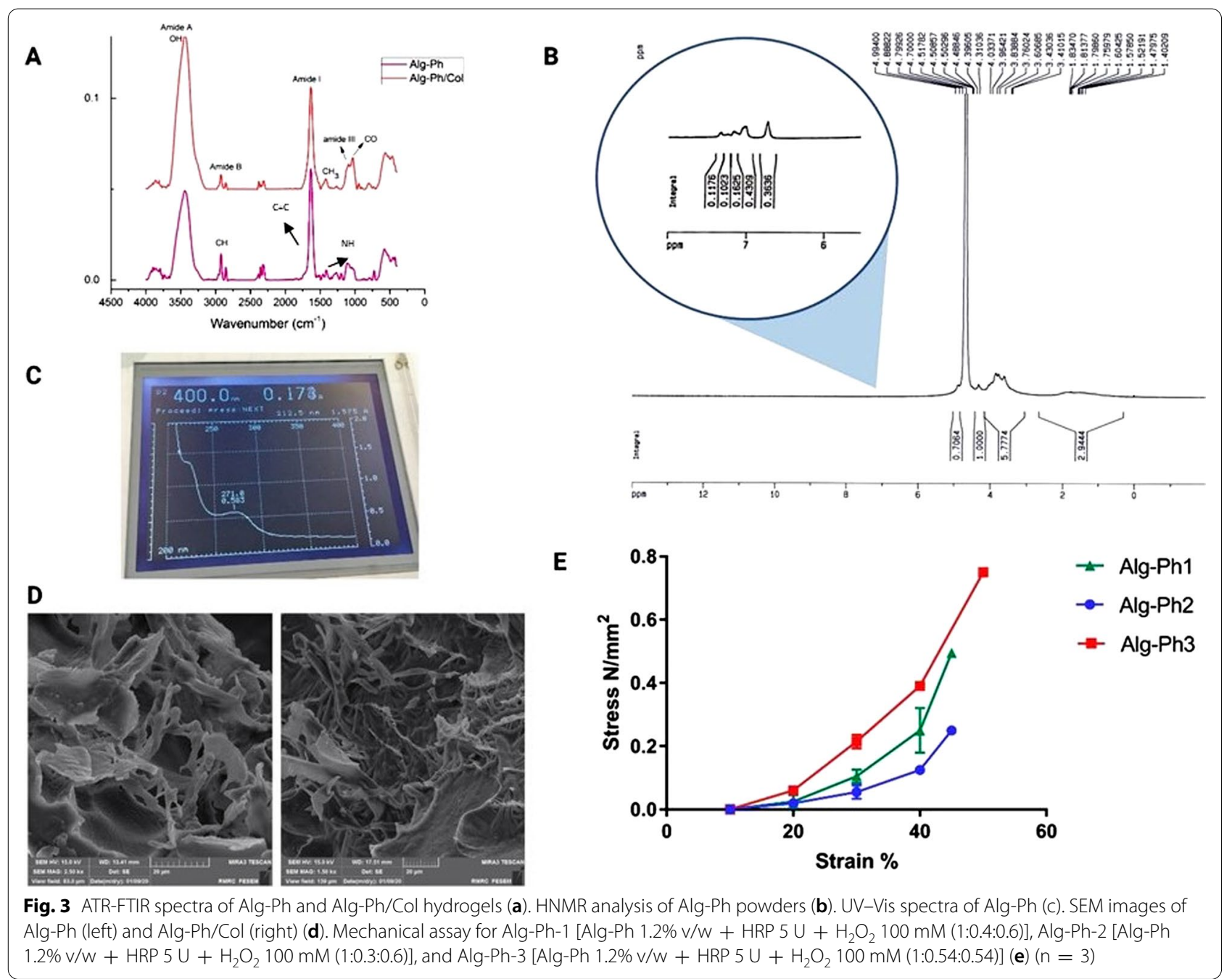

an incredible factor for hydrogel evaluation [21]. Data showed that the longer gelation times result from the enhanced concentration of $\mathrm{H}_{2} \mathrm{O}_{2}$ and decreased concentration of HRP which can be explained as a consequence of the deactivation of HRP by $\mathrm{H}_{2} \mathrm{O}_{2}$. To select a composition based on appropriate gelation time (as an optimizing factor), several items including mechanical and morphological properties should be considered. On the other hand, it should be noted that $\mathrm{H}_{2} \mathrm{O}_{2}$ is a strong oxidant with a damaging effect; a decreased gelation time with a lower concentration of $\mathrm{H}_{2} \mathrm{O}_{2}$ is an appropriate chemical agent for biological applications. It should be considered that in the HRP-mediated cross-linking method, Ph derivatives are used because of electron donor capacity. In this reaction, $\mathrm{HRP}$ reacts with $\mathrm{H}_{2} \mathrm{O}_{2}$ quickly, and the consequential compound can oxidize phenolic hydroxyl $(\mathrm{Ph})$ groups [16]. Therefore, another important factor that influences the gelation time is the level of Ph groups in Alg-based hydrogel. We examined three different concentrations of Alg- $\mathrm{Ph} 1,1.2$, and 1.5\% (v/w) and monitored gelation time. Figure 3 shows that the lowest value for gelation time is achievable at the concentration of $1.2 \%$. The elevation of Alg-Ph concentration led to delayed gelation time. This effect is associated with the existence of excessive amounts of unreacted Ph groups and can produce soft gels with extremely low mechanical stability. We found that the addition of Col to the Alg- $\mathrm{Ph}$ hydrogel increases the gelation time. It is suggested that the distribution of Col microfibrils inside the hydrogel supports platform to react with Alg- $\mathrm{Ph}$ molecules thus limits the availability of $\mathrm{Ph}$ groups to $\mathrm{HRP} / \mathrm{H}_{2} \mathrm{O}_{2}$. After several technical replicates, we found that hydrogel composed of $1.2 \%(\mathrm{w} / \mathrm{v}) \mathrm{Alg}-\mathrm{Ph}, 5(\mathrm{u} / \mathrm{ml}) \mathrm{HRP}$, and $100(\mathrm{mM})$ $\mathrm{H}_{2} \mathrm{O}_{2}$ exhibited appropriate stability for fabrication of cartilage-like engineered scaffold. To find the final concentration, three compositions were selected and investigated by mechanical testing. The results showed that the Alg-Ph3 hydrogel had the highest ultimate strength, 
compressive modulus, and strain in comparison with Alg-Ph 1 and 2 groups. In the optimized values, HRP and $\mathrm{H}_{2} \mathrm{O}_{2}$ efficiently interact with each other in the presence of Ph groups.

\section{Conclusion}

Alg is a versatile polymer that can be used for an extensive range of biomedical applications. Enzymatic crosslinking of Alg improves the flexibility of the final hydrogel and yielded favorable mechanical properties. We found that mechanical and physical properties of $1.2 \%(\mathrm{v} / \mathrm{w})$ Alg-Ph, $5 \mathrm{U}$ HRP, $100 \mathrm{mM} \mathrm{H}_{2} \mathrm{O}_{2}$ at the ratio of 1:0.54; 0.54 are appropriate for cartilage-like engineered scaffolds.

\section{Limitation}

This work faces numerous limitations. Further investigations are needed to assess different concentrations of hydrogel $\mathrm{H}_{2} \mathrm{O}_{2}$, HRP, and Alg-Ph. It is suggested to monitor the gelation time and mechanical properties of Alg-based hydrogels after the addition of other ECM components. Besides, we did not examine the developed hydrogel in in vivo conditions. It should not be forgotten that the flexibility and consistency of Alg-Ph-based hydrogel can be different inside the body compared to the in vitro conditions.

\begin{abstract}
Abbreviations
EDC: 1-Ethyl-3-(3-dimethylaminopropyl) carbodiimide; MES: 2-Morpholinoethanesulfonic acid, monohydrate; Alg: Alginate; Col: Collagen; EtOH: Ethanol; ATR-FTIR: Fourier transform infrared spectroscopy; HRP: Horseradish peroxidase; $\mathrm{H}_{2} \mathrm{O}_{2}$ : Hydrogen peroxide; NHS: N-hydroxysuccinimide; NMR: Nuclear magnetic resonance; Ph: Phenol; SEM: Scanning electron microscopy; UV-Vis: Ultraviolet-visible spectroscopy.
\end{abstract}

\section{Acknowledgements}

Authors wish to thank the personnel of the Faculty of Advanced Medical Sciences and Stem Cell Research Center for help and guidance.

\section{Authors' contributions}

SS, ABK, HTN, RR and LR performed the experiments. ABK and RR supervised the study. All authors read and approved the final manuscript.

\section{Funding}

This study was supported by a Grant (IR.TBZMED.VCR.REC.1397.238) from Tabriz University of Medical Sciences.

\section{Availability of data and materials}

The data created and analyzed during the current study are available from the corresponding author upon reasonable requests.

\section{Declarations}

Ethics approval and consent to participate

Not applicable.

\section{Consent for publication}

Not applicable.

\section{Competing interests}

Authors declare that they have a competing interests.

\section{Author details}

${ }^{1}$ Department of Tissue Engineering, Faculty of Advanced Medical Sciences, Tabriz University of Medical Sciences, Tabriz, Iran. ${ }^{2}$ Chemical Engineering Faculty, Sahand University of Technology, 51335-1996 Tabriz, Iran. ${ }^{3}$ Stem Cell Research Center, Tabriz University of Medical Sciences, Tabriz, Iran. ${ }^{4}$ Department of Applied Cell Sciences, Faculty of Advanced Medical Sciences, Tabriz University of Medical Sciences, 5154853431 Tabriz, Iran.

Received: 19 June 2021 Accepted: 20 September 2021

Published online: 28 September 2021

\section{References}

1. Farokhi M, Jonidi Shariatzadeh F, Solouk A, Mirzadeh H. Alginate based scaffolds for cartilage tissue engineering: a review. Int J Polym Mater Polym Biomater. 2020;69(4):230-47.

2. Choi M-Y, Kim J-T, Lee W-J, Lee Y, Park KM, Yang Y-I, Park KD. Engineered extracellular microenvironment with a tunable mechanical property for controlling cell behavior and cardiomyogenic fate of cardiac stem cells. Acta Biomater. 2017;50:234-48.

3. Kwon S-H, Choi J-Y, Ahn GY, Jang WS, Shin J-W, Na J-I, Park K-C, Huh C-H. The efficacy and safety of microneedle monopolar radiofrequency for the treatment of periorbital wrinkles. J Dermatol Treat. 2019. https://doi.org/ 10.1080/09546634.2019.1662880.

4. Buwalda SJ, Vermonden T, Hennink WE. Hydrogels for therapeutic delivery: current developments and future directions. Biomacromol. 2017;18(2):316-30.

5. Chuah YJ, Peck Y, Lau JEJ, Hee HT, Wang D-A. Hydrogel based cartilaginous tissue regeneration: recent insights and technologies. Biomater Sci. 2017;5(4):613-31.

6. Kurisawa M, Lee F, Wang L-S, Chung JE. Injectable enzymatically crosslinked hydrogel system with independent tuning of mechanical strength and gelation rate for drug delivery and tissue engineering. J Mater Chem. 2010;20(26):5371-5.

7. Tibbitt MW, Anseth KS. Hydrogels as extracellular matrix mimics for $3 \mathrm{D}$ cell culture. Biotechnol Bioeng. 2009;103(4):655-63.

8. Khanmohammadi M, Dastjerdi MB, Ai A, Ahmadi A, Godarzi A, Rahimi A, Ai J. Horseradish peroxidase-catalyzed hydrogelation for biomedical applications. Biomater Sci. 2018;6(6):1286-98.

9. Sakai S, Kawakami K. Synthesis and characterization of both ionically and enzymatically cross-linkable alginate. Acta Biomater. 2007;3(4):495-501.

10. Teixeira LSM, Feijen J, van Blitterswijk CA, Dijkstra PJ, Karperien M. Enzyme-catalyzed crosslinkable hydrogels: emerging strategies for tissue engineering. Biomaterials. 2012;33(5):1281-90.

11. Gantumur E, Sakai S, Nakahata M, Taya M. Cytocompatible enzymatic hydrogelation mediated by glucose and cysteine residues. ACS Macro Lett. 2017;6(5):485-8.

12. Condello V, Filardo G, Madonna V, Andriolo L, Screpis D, Bonomo M, Zappia M, Dei Giudici L, Zorzi C. Use of a biomimetic scaffold for the treatment of osteochondral lesions in early osteoarthritis. Biomed Res Int. 2018;2018:7937089. https://doi.org/10.1155/2018/7937089.

13. Yang X, Lu Z, Wu H, Li W, Zheng L, Zhao J. Collagen-alginate as bioink for three-dimensional (3D) cell printing based cartilage tissue engineering. Mater Sci Eng C. 2018;83:195-201.

14. Kurisawa M, Chung JE, Yang YY, Gao SJ, Uyama H. Injectable biodegradable hydrogels composed of hyaluronic acid-tyramine conjugates for drug delivery and tissue engineering. Chem Commun. 2005;34:4312-4.

15. Poolman JM, Boekhoven J, Besselink A, Olive AG, Van Esch JH, Eelkema R. Variable gelation time and stiffness of low-molecular-weight hydrogels through catalytic control over self-assembly. Nat Protoc. 2014;9(4):977-88.

16. Sakai S, Yamada Y, Zenke T, Kawakami K. Novel chitosan derivative soluble at neutral pH and in-situ gellable via peroxidase-catalyzed enzymatic reaction. J Mater Chem. 2009;19(2):230-5.

17. Saghati S, Rahbarghazi R, Baradar Khoshfetrat A, Moharamzadeh K, Tayefi Nasrabadi H, Roshangar L. Phenolated alginate-collagen hydrogel induced chondrogenic capacity of human amniotic mesenchymal stem cells. J Biomater Appl. 2021. https://doi.org/10.1177/08853282211021692.

18. Ahmadian M, Khoshfetrat AB, Khatami N, Morshedloo F, Rahbarghazi R, Hassani A, Kiani S. Influence of gelatin and collagen incorporation on 
peroxidase-mediated injectable pectin-based hydrogel and bioactivity of fibroblasts. J Biomater Appl. 2020. https://doi.org/10.1177/0885328220 977601.

19. Belbachir K, Noreen R, Gouspillou G, Petibois C. Collagen types analysis and differentiation by FTIR spectroscopy. Anal Bioanal Chem. 2009;395(3):829-37.

20. Sakai S, Khanmohammadi M, Khoshfetrat AB, Taya M. Horseradish peroxidase-catalyzed formation of hydrogels from chitosan and poly (vinyl alcohol) derivatives both possessing phenolic hydroxyl groups. Carbohyd Polym. 2014;111:404-9.
21. Khatami N, Khoshfetrat AB, Khaksar M, Zamani ARN, Rahbarghazi R. Collagen-alginate-nano-silica microspheres improved the osteogenic potential of human osteoblast-like MG-63 cells. J Cell Biochem. 2019;120(9):15069-82.

\section{Publisher's Note}

Springer Nature remains neutral with regard to jurisdictional claims in published maps and institutional affiliations.
Ready to submit your research? Choose BMC and benefit from:

- fast, convenient online submission

- thorough peer review by experienced researchers in your field

- rapid publication on acceptance

- support for research data, including large and complex data types

- gold Open Access which fosters wider collaboration and increased citations

- maximum visibility for your research: over $100 \mathrm{M}$ website views per year

At BMC, research is always in progress.

Learn more biomedcentral.com/submissions 